\title{
Correction to: Geographic variation in thermal tolerance and morphology in a fiddler crab sister-species pair
}

\author{
M. Zachary Darnell ${ }^{1,2} \cdot$ Kelly M. Darnell ${ }^{3,4}$
}

Published online: 6 February 2020

c) Springer-Verlag GmbH Germany, part of Springer Nature 2020

\section{Correction to: Marine Biology (2018) 165:26 \\ https://doi.org/10.1007/s00227-017-3282-y}

In the original published article, the separators between numerator and denominator degrees of freedom in Tables 2 and 3 were excluded. The corrected Tables 2 and 3 are as follows.
Table 2 Results of ANCOVA analyses of $C T_{\max }$

\begin{tabular}{llllll}
\hline Source & DF & SS & $F$ & $P$ & $\eta_{\text {partial }}^{2}$ \\
\hline Full model & $3 / 137$ & 5.409 & 9.965 & $<0.0001$ & - \\
Species & 1 & 0.178 & 0.985 & 0.323 & 0.007 \\
Summer max. temp. & 1 & 1.199 & 6.627 & 0.011 & 0.046 \\
$\begin{array}{l}\text { Species } \times \text { summer max. } \\
\text { temp. }\end{array}$ & 1 & 0.319 & 1.766 & 0.186 & 0.013 \\
\hline
\end{tabular}

The original article can be found online at https://doi.org/10.1007/ s00227-017-3282-y.

\section{Zachary Darnell}

zachary.darnell@usm.edu

1 Division of Coastal Sciences, School of Ocean Science and Technology, The University of Southern Mississippi, 703 E. Beach Dr, Ocean Springs, MS 39564, USA

2 Department of Biological Sciences, Nicholls State University, Thibodaux, LA 70310, USA

3 The Water Institute of the Gulf, Baton Rouge, LA 70825, USA

4 Present Address: Division of Coastal Sciences, School of Ocean Science and Technology, The University of Southern Mississippi, Ocean Springs, MS 39564, USA 
Table 3 Results of analysis of morphology by species and long-term average temperature

\begin{tabular}{llrrrc}
\hline Source & DF & \multicolumn{1}{l}{ SS } & \multicolumn{1}{l}{ F } & $P$ & $\eta_{\text {partial }}^{2}$ \\
\hline Carapace width & & & & & \\
$\quad$ Full model & $3 / 412$ & 720.442 & 80.825 & $<0.0001$ & - \\
$\quad$ Species & 1 & 47.033 & 15.830 & $<0.0001$ & 0.037 \\
Winter min. temp. & 1 & 0.101 & 0.034 & 0.854 & 0.0001 \\
$\quad$ Species $\times$ winter min. temp. & 1 & 533.238 & 179.470 & $<0.0001$ & 0.303 \\
Relative claw length & & & & & \\
$\quad$ Summer max. temp. (L. pugilator) & $1 / 187$ & 52.281 & 23.379 & $<0.0001$ & 0.111 \\
Average temp. (L. panacea) & $1 / 225$ & 19.672 & 11.827 & $<0.001$ & 0.499 \\
\hline
\end{tabular}

Carapace width was analyzed using an ANCOVA, while relative claw length was analyzed using separate linear regressions for each species

Publisher's Note Springer Nature remains neutral with regard to jurisdictional claims in published maps and institutional affiliations. 